\title{
Elevação dos níveis de CA 19-9 em portadores de Síndrome de Mirizzi na ausência de doença maligna: um relato de caso
}

\author{
Elevation of CA 19-9 levels in patients with Mirizzi Syndrome in the absence of malignant \\ disease: a case report
}
Elevación de CA 19-9 en pacientes con Síndrome de Mirizzi en ausencia de enfermedad maligna: Informe de un caso

Rodolpho Cesar Oliveira Mellem Kairala ${ }^{1 *}$, Paula Ananda Chacon Inês ${ }^{2}$, Rafaella Gregori Perduca ${ }^{2}$, Bruna Lima Daher ${ }^{1}$, Bruna Lemos Silva ${ }^{1}$, Afranio Faria Lemos ${ }^{1}$, Danilo Rocha Chavez Zambrana ${ }^{1}$, Caio Cesar Faciroli Contin Silva ${ }^{1}$, Cláudio Henrique Formigoni Reviriego ${ }^{1}$, Maria Clara Nobrega Pereira $^{1}$

\section{RESUMO}

Objetivo: Descrever o caso raro de elevação dos níveis de CA 19-9 na presença de Síndrome de Mirizzi, uma afecção benigna do trato biliar, sem relação com a presença de doença maligna. Relato de caso: Mulher, 61 anos, admitida ao serviço de emergência com história de dor abdominal em epigástrio, com irradiação para hipocôndrio direito e dorso, associada a episódios de náuseas e vômitos, além de quadro de icterícia com colúria e acolia fecal. Durante investigação diagnóstica apresentava CA 19-9 de 8.962U/mL, foi realizada colangioressonância, a qual evidenciou imagem sugestiva de síndrome de Mirizzi. Paciente submetida colecistectomia videolaparoscópica associada a colangiopancreatografia retrógrada com passagem de dreno plástico intra-colédoco, apresentando intensa drenagem de conteúdo biliar para duodeno. No seguimento, paciente permaneceu sem intercorrências ou evidências de doença maligna, com redução dos níveis séricos de CA 19-9 para 28,9U/mL após 12 semanas da cirurgia. Considerações finais: A síndrome de Mirizzi, ainda que pouco frequente, é uma das possibilidades etiológicas para a elevação do marcador tumoral CA 19-9. É um diagnóstico benigno para situações que cursam com elevação do antígeno carboidrato de superfície celular, que tem seu valor de referência normalizado após tratamento cirúrgico do processo obstrutivo.

Palavras-chave: Síndrome de Mirizzi, Antígeno CA-19-9, Hiperbilirrubinemia.

\begin{abstract}
Objective: To describe the rare case of elevated CA 19-9 levels in the presence of Mirizzi Syndrome, a benign biliary tract disorder, unrelated to the presence of malignant disease. Case report: A 61-year-old woman was admitted to the emergency department with a history of abdominal pain in epigastrium, with irradiation to the right hypochondria and back, associated with episodes of nausea and vomiting, as well as jaundice with choluria and fecal acolia. During diagnostic investigation, CA $19-9$ of $8,962 \mathrm{U} / \mathrm{mL}$, cholangio-resonance was performed, which showed an image suggestive of Mirizzi syndrome. Patient who underwent videolaparoscopic cholecystectomy associated with retrograde cholangiopancreatography with the passage of an intracholedochal plastic drain, presenting intense drainage of biliary content to the duodenum. At follow-up, the patient remained uneventful or had evidence of malignant disease, with a reduction in serum CA 19-9 to $28.9 \mathrm{U} / \mathrm{mL}$ after 12 weeks of surgery. Final considerations: Although uncommon, Mirizzi syndrome is one of
\end{abstract}

1 Santa Casa de Misericórdia de Franca, Franca - SP. *E-mail: rodskairala@gmail.com

2 Uni-FACEF Centro Universitário Municipal de Franca - SP. 
the etiological possibilities for the elevation of tumor marker CA 19-9. It is a benign diagnosis for situations involving elevated cell surface carbohydrate antigen, which has its normalized reference value after surgical treatment of the obstructive process.

Keywords: Mirizzi Syndrome, CA-19-9 Antigen, Hyperbilirubinemia.

\section{RESUMEN}

Objetivo: Describir el caso raro de niveles elevados de CA 19-9 en presencia del síndrome de Mirizzi, un trastorno benigno del tracto biliar, no relacionado con la presencia de enfermedad maligna. Caso clínico: Una mujer de 61 años ingresó en el servicio de urgencias con antecedentes de dolor abdominal en el epigastrio, con irradiación hacia la hipocondría derecha y la espalda, asociada con episodios de náuseas y vómitos, así como ictericia con coluria y acolia fecal. Durante la investigación diagnóstica, se realizó CA 19-9 de $8,962 \mathrm{U} / \mathrm{mL}$, colangio-resonancia, que mostró una imagen sugestiva del síndrome de Mirizzi. Paciente sometido a colecistectomía videolaparoscópica asociada a colangiopancreatografía retrógrada con drenaje plástico intracoledocal, que presenta un drenaje intenso del contenido biliar al duodeno. En el seguimiento, el paciente permaneció sin incidentes o tenía evidencia de enfermedad maligna, con una reducción en el suero de CA 19-9 a 28.9U/mL después de 12 semanas de cirugía. Consideraciones finales: El síndrome de Mirizzi, aunque poco frecuente, es una de las posibilidades etiológicas para la elevación del marcador tumoral CA 199. Es un diagnóstico benigno para situaciones que involucran un antígeno de carbohidrato de superficie celular elevado, que tiene su valor de referencia normalizado después del tratamiento quirúrgico del proceso obstructivo.

Palabras clave: Síndrome de Mirizzi, Antígeno CA-19-9, Hiperbilirrubinemia.

\section{INTRODUÇÃO}

A síndrome de Mirizzi é uma afecção que decorre de um cálculo biliar impactado no ducto cístico ou no colo da vesícula biliar, ocasionando inflamação e obstrução do ducto hepático comum ou do ducto colédoco por uma compressão extrínseca.

História de icterícia recorrente, presença dos elementos que compõem a tríade de Charcot (icterícia, dor no hipocôndrio direito e febre com calafrios) associados a quadros de náuseas e/ou vômitos, a persistência de níveis elevados de enzimas canaliculares, principalmente a fosfatase alcalina, associados a achados ultrassonográficos, como vesícula biliar contraída, constituem os elementos sugestivos desta síndrome, porém o seu diagnóstico é feito no pré-operatório em apenas $5 \%$ dos casos (GIBOR U, et al., 2015; LEITE LMC e COELHO JF, 2006; SHAH OJ, et al., 2001; SILVA JB, et al., 2016).

A pesquisa diagnóstica deve incluir exames como ultrassom de abdome, tomografia computadorizada de abdome, colangiografia transparietohepática ou colangiopancreatografia endoscópica retrógrada (CPRE). O tratamento cirúrgico é complicado e controverso na literatura, pois a inflamação vesicular e perivesicular, resultante da impactação de cálculos no ducto cístico ou infundíbulo vesicular, dificulta a identificação das estruturas anatómicas, implicando maior risco de lesão iatrogênica da via biliar durante o ato cirúrgico, por isso o diagnóstico pré-operatório da síndrome de Mirizzi se faz tão importante (JUNIOR EVM, et al., 2015; ENGLAND RE e MARTIN DF, 2017).

A colecistectomia, convencional ou videolaparoscópica - a depender da experiência do cirurgião, é usualmente resolutiva na ausência de fístula. Caso haja fístula, o tratamento cirúrgico deve incluir o reparo das lesões e, em certos casos, o uso de próteses biliares, que podem ser aplicadas por cirurgia aberta coledocotomia - ou via CPRE (JUNIOR EVM, et al., 2015).

A CPRE é a técnica de eleição para estabelecer o diagnóstico de fístula, permitindo uma boa definição anatómica da via biliar, além disso, pode ser também terapêutica, permitindo uma abordagem não cirúrgica, particularmente importante nos maus candidatos cirúrgicos e nos casos de colangite, em que a 
descompressão prévia da via biliar é essencial. Contudo, a resolução completa da síndrome de Mirizzi sem recurso à cirurgia é rara (ENGLAND RE e MARTIN DF, 2017).

Pablo Luis Mirizzi, em 1948, foi o primeiro a descrever a síndrome de Mirizzi a partir da observação de situações que poderiam causar colestase extra-hepática em grupos determinados de portadores de colelitíase. No início dos estudos, Pablo justificou a fisiopatologia da síndrome com a existência de mecanismo esfincteriano presente no hepatocolédoco. Nos dias de hoje já se tem o conhecimento de que não há esfíncter, contudo, mesmo depois que descoberto a real causa, manteve-se o epônimo da síndrome como Mirizzi (LAI ECH e LAU WY, et al., 2006).

A síndrome de Mirizzi é uma complicação obstrutiva benigna e rara da colecistolitíase. Por meio de estudos epidemiológicos já foi constatado uma maior prevalência no sexo feminino com idade entre 21 e 90 anos, o que pode ser justificado pela preponderância de litíase biliar neste grupo. Além disso, sabe-se que há uma prevalência de $0,05 \%$ a $4 \%$ de ocorrência da síndrome de Mirizzi em portadores de colelitíase, sendo considerada uma complicação de colecistolitíase de longa data (IBRARULLAH M, et al., 2008; LACERDA PS, et al., 2014).

Atualmente subdivide-se a patologia de acordo com o grau de compressão que a mesma exerce no ducto hepático ou a presença de fístulas e o diagnóstico diferencial deve ser feito com doenças como coledocolitíase e doenças periampulares, incluindo o câncer de pâncreas, patologias que também ocasionam quadros de icterícia obstrutiva (GIBOR U, et al., 2015).

A nova classificação da síndrome de Mirizzi é baseada em tipos enumerados de I a $\mathrm{V}$, sendo que nesta última há subdivisão em A e B. No tipo I é detectado uma compressão extrínseca do ducto hepático comum/colédoco por cálculo no colo vesicular ou ducto cístico; a medida que se tem a presença de fístula colecistobiliar de diâmetro inferior a $1 / 3$ da circunferência do ducto hepático comum/colédoco, tem como classificação tipo II; se o diâmetro da fístula for superior a 2/3 da circunferência classifica-se como tipo III; e se esta fístula envolver toda a circunferência já é tipo IV. A partir do momento em que a fístula se torna colecistoentérica, a classificação passa a ser tipo V, podendo ser subdividida em $\mathrm{A}$ se não houver íleo biliar ou B se tem a presença de íleo biliar (CSENDES A., et al., 2008).

Em grande parte dos casos, para a diferenciação de afecções benignas das afecções malignas, como o câncer de pâncreas, é utilizada a análise do antígeno carboidrato 19-9 (CA 19-9), um antígeno de carboidrato sérico identificado por um anticorpo monoclonal, sendo descrito inicialmente seu aparecimento contra células de uma linhagem do carcinoma colorretal (FONTES PRO, et al., 2012).

É um marcador tumoral comumente utilizado para diagnóstico e acompanhamento de doenças malignas do trato gastrointestinal, especialmente pancreático-biliar, todavia a presença de hiperbilirrubinemia torna-se um fator de confusão significativo, visto que sua síntese acontece por células epiteliais normais das vias biliares e células tumorais, sendo excretado na bile, possibilitando o aumento dos níveis de CA 19-9 em casos de obstrução biliar benigna e maligna, uma vez que ocorre refluxo deste para a corrente sanguínea secundário a estase biliar, contudo valores de CA $19-9$ superiores a $1.000 \mathrm{U} / \mathrm{mL}$ são raros em doenças benignas (BALLEHANINNA UK e CHAMBERLAIN RS, 2012; GIBOR U, et al., 2015; ROBERTSON AG e DAVIDSON BR, 2007).

O presente estudo tem como objetivo relatar um caso raro em que um paciente do sexo feminino apresentou uma elevação dos níveis de CA 19-9 na presença de Síndrome de Mirizzi, uma afecção benigna do trato biliar, sem relação com a presença de doença maligna e realizar uma breve e importante revisão de literatura sobre o assunto.

\section{RELATO DO CASO}

Paciente do sexo feminino, de 61 anos, foi admitida ao serviço de emergência, da equipe de cirurgia geral da Fundação Santa Casa de Misericórdia de Franca, com história de dor abdominal em região epigástrica, com irradiação para região de hipocôndrio direito e dorso, iniciada há quatro dias da procura por atendimento médico. A dor era classificada pela paciente como moderada à forte intensidade, associada a episódios de 
náuseas e vômitos, além de quadros de icterícia progressiva com colúria e acolia fecal. Ao exame físico apresentava icterícia moderada, com abdome doloroso à palpação de epigástrio e hipocôndrio direito sem sinais de irritação peritoneal, sinal de Murphy - o qual consiste em provocar dor pela palpação da vesícula biliar, durante a inspiração profunda - negativo e ausência de visceromegalias ou massas palpáveis.

Durante admissão foram realizados exames laboratoriais (Tabela 1) que permitiram confirmar 0 diagnóstico sindrômico de síndrome colestática, afastando a possibilidade de pancreatite biliar devidos resultados de amilase e lipase normais.

Tabela 1 - Resultados laboratoriais coletados na admissão.

\begin{tabular}{|c|c|c|}
\hline Exames & Referências & Resultados \\
\hline Hemoglobina $(\mathrm{Hb})-\mathrm{g} / \mathrm{dL}$ & $11,5-14,9$ & 12 \\
\hline Hematócrito $(\mathrm{Ht})-\%$ & $35,9-44,6$ & 33,8 \\
\hline Plaquetas $(\mathrm{Pq})-/ \mathrm{mm}^{3}$ & $150.000-450.000$ & 230.000 \\
\hline Leucócitos (Lc) - /mm³ & $4.400-11.000$ & 11.900 \\
\hline Bastonetes (Bt) - \% & $\sim 3$ & 4 \\
\hline Segmentados $(\mathrm{Sg})-\%$ & $\sim 56$ & 77,2 \\
\hline Linfócitos (Lf) - \% & $\sim 34$ & 10,3 \\
\hline Bilirrubina total $(\mathrm{BT})-\mathrm{mg} / \mathrm{dL}$ & $0,3-1$ & 11 \\
\hline Bilirrubina Direta $(\mathrm{BD})-\mathrm{mg} / \mathrm{dL}$ & $\leq 0,4$ & 7,7 \\
\hline $\begin{array}{c}\text { Gama Glutamil-transferase (GGT) } \\
\qquad U / L\end{array}$ & $8-41$ & 366 \\
\hline Fosfatase Alcalina $(F A)-U / L$ & $50-120$ & 235 \\
\hline $\begin{array}{l}\text { Transaminase glutâmico- } \\
\text { oxalacética (TGO) - U/L }\end{array}$ & $15-30$ & 164 \\
\hline $\begin{array}{l}\text { Transaminase glutâmico-pirúvica } \\
\qquad(T G P)-U / L\end{array}$ & $8-35$ & 238 \\
\hline Amilase - U/L & $20-300$ & 44 \\
\hline Lipase - U/L & $<200$ & 58,6 \\
\hline Creatinina $(\mathrm{Cr})-\mathrm{mg} / \mathrm{dL}$ & $\leq 1,1$ & 1,3 \\
\hline Uréia (Ur) - mg/dL & $15-36$ & 31 \\
\hline
\end{tabular}

Fonte: Kairala RCOM, et al., 2019. Adaptado de Rosenfeld LG, et al., 2019.

Após 4 dias de internação foram realizados novos exames (Tabela 2), evidenciando progressão do quadro colestático. Dos exames realizados, foi dado uma importância maior ao CA 19-9, o qual apresentou um valor consideravelmente elevado, sugestivo de neoplasia periampular. 
Tabela 2 - Resultados laboratoriais coletados durante internação.

\begin{tabular}{lll}
\hline Exames & Referências & Resultado \\
\hline $\mathrm{Hb}-\mathrm{g} / \mathrm{dL}$ & $11,5-14,9$ & 11,8 \\
$\mathrm{Ht}-\%$ & $35,9-44,6$ & 33 \\
$\mathrm{Pq}-/ \mathrm{mm}^{3}$ & $150.000-450.000$ & 390.000 \\
$\mathrm{Lc}-/ \mathrm{mm}^{3}$ & $4.400-11.000$ & 15.100 \\
$\mathrm{Bt}-\%$ & $\sim 3$ & 14 \\
$\mathrm{Sg}-\%$ & $\sim 56$ & 63,2 \\
$\mathrm{Lf}-\%$ & $\sim 34$ & 12,4 \\
$\mathrm{BT}-\mathrm{mg} / \mathrm{dL}$ & $0,3-1$ & 17,7 \\
$\mathrm{BD}-\mathrm{mg} / \mathrm{dL}$ & $\leq 0,4$ & 12,7 \\
$\mathrm{GGT}-\mathrm{U} / \mathrm{L}$ & $8-41$ & 545 \\
$\mathrm{FA}-\mathrm{U} / \mathrm{L}$ & $50-120$ & 354 \\
$\mathrm{TGO}-\mathrm{U} / \mathrm{L}$ & $15-30$ & 188 \\
$\mathrm{TGP}-\mathrm{U} / \mathrm{L}$ & $8-35$ & 229 \\
$\mathrm{Cr}-\mathrm{mg} / \mathrm{dL}$ & $20-300$ & 1,0 \\
$\mathrm{Ur}-\mathrm{mg} / \mathrm{dL}$ & $<200$ & 27 \\
Antígeno Carcinoembrionário (CEA) - ng/dL & $0-3$ & 3,11 \\
Alfa-fetoproteína - ng/dL & Tabagistas: $3,8-5,5$ & 1,5 \\
Antígeno Carboidrato 19-9 (CA 19-9) - U/mL & $<15$ & 8.962 \\
\hline
\end{tabular}

Fonte: Kairala RCOM, et al., 2019. Adaptado de Rosenfeld LG, et al., 2019.

Diante da hipótese de tumor periampular, foram solicitadas ultrassonografia (US) de abdome total e tomografia computadorizada (TC) de abdome, que identificaram dilatação das vias biliares extra e intrahepáticas, com colédoco medindo $1,0 \mathrm{~cm}$, além de vesícula biliar com paredes espessadas, contendo cálculos.

Após os resultados dos exames de imagem, realizou-se endoscopia digestiva alta (EDA) para avaliação da papila duodenal, que não demonstrou alterações em região periampular, e colangioressonância (CR), a qual evidenciou cálculo em topografia da junção do ducto cístico com o terço médio/proximal do colédoco, determinando pequena a moderada dilatação das vias biliares extra e intra-hepáticas à montante, sugestivo de Síndrome de Mirizzi.

Por meio dos achados da CR, optou-se por realizar colecistectomia videolaparoscópica associada a colangiopancreatografia retrógrada endoscópica (CPRE) com passagem de prótese biliar plástica intracolédoco, devido presença de fístula colecistobiliar tipo II. Após passagem de prótese, observou-se intensa drenagem de contraste e conteúdo biliar para duodeno.

Paciente evoluiu bem no pós-operatório, com redução dos marcadores de colestase. No seguimento paciente permaneceu em bom estado geral, sem intercorrências ou evidências de doença maligna. $O$ relatório anatomopatológico de vesícula biliar não evidenciou malignidade, sendo identificado apenas colecistite crônica inespecífica, paciente também apresentou, após 8 semanas de pós-operatório, redução dos níveis séricos de CA 19-9 para 28,9 U/mL, atingindo a normalidade. Paciente seguiu em acompanhamento médico mensal até o sexto mês de pós-operatório, se mantendo assintomática, sem alterações clínicas ou laboratoriais.

\section{DISCUSSÃO}


A síndrome de Mirizzi foi descrita primeiramente no ano de 1948 por Pablo Luis Mirizzi. Atualmente é classificada em cinco tipos, sendo usado como parâmetros a compressão do ducto hepático ou a existência de fístula colecistobiliar ou colecistoentérica e o diâmetro desta.

Este tipo de acometimento pode ser sugerido através de alguns exames, como os de imagem da via biliar, contudo há uma dificuldade no diagnóstico pré-operatório e muitos pacientes acabam não sendo diagnosticados e desta forma correndo maior risco de lesão da via biliar, de forma iatrogênica, durante 0 ato cirúrgico (LAI ECH e LAU WY, et al., 2006; PRINCIPE A, et al., 2003; CSENDES A., et al., 2008).

A compressão extrínseca da via biliar devido à impactação de cálculos no ducto cístico ou no infundíbulo da vesícula, cursa geralmente com um quadro de dor abdominal associada a icterícia e colangite. Além desses sintomas, o paciente pode apresentar náuseas, vômitos, colúria, acolia fecal, prurido e hepatomegalia, sendo que o diagnóstico é dado na maior parte dos casos, através de exames de imagem (LIN CL, et al., 1997; FONTES PRO, et al., 2012).

A alteração de exames como o CA 19-9 em portadores de doenças benignas possui relatos na literatura e pode surgir em pacientes com doenças biliopancreáticas ou em outros locais do trato gastrointestinal, todavia, a elevação em níveis muito altos é incomum nas patologias benignas. A síndrome de Mirizzi apresenta-se como uma das condições raras que podem ter essa elevação dos níveis de CA 19-9, sendo está a situação aqui relatada (FONTES PRO, et al., 2012).

Muitos casos benignos que apresentam elevação dos níveis do CA 19-9 surgem diante de um quadro de hiperbilirrubinemia secundária à obstrução biliar e os valores deste antígeno tem como característica decrescerem ou até normalizarem depois que se tem a resolução do quadro colestático. Este fato foi evidenciado no quadro da paciente aqui relatada, a qual já apresentava normalização desse marcador após 8 semanas de pós-operatório (MANN DV, et al., 2000; ROBERTSON AG e DAVIDSON BR, 2007).

Hodiernamente, sabe-se que o antígeno marcador CA 19-9 é um produto produzido pelo epitélio da via biliar e secretado por meio da bile. Desta forma, diante de uma obstrução em um ponto da via biliar, tem-se como consequência uma diminuição da excreção deste produto e, consequentemente, aumento na sua absorção, o que contribui para o seu achado alterado nos exames laboratoriais.

Em patologias benignas, após melhora do quadro, seus valores tender a se normalizar devido ao retorno da fisiologia normal da via biliar, o que é raro nas doenças malignas, uma vez que a neoplasia existente que é a responsável pela produção do antígeno (MANN DV, et al., 2000).

Por meio de todas evidências científicas, sabe-se que a elevação do marcador CA 19-9 não indica de maneira absoluta a malignidade ou a benignidade de uma patologia e, portanto, não pode ser usado como indicador único para diferenciação entre essas duas entidades.

A síndrome de Mirizzi apresenta-se como um exemplo das patologias benignas que precisam ser lembradas para se fazer o diagnóstico diferencial. Contudo, sabe-se que diante de paciente com elevação dos níveis de CA 19-9 e quadro clínico de síndrome colestática, o primeiro diagnóstico que deve ser investigado é o de malignidade, para que este seja confirmado ou descartado e assim, neste último caso, iniciar a investigação dos demais diagnósticos (SANCHEZ M, et al., 2006).

\section{CONSIDERAÇÕES FINAIS}

A síndrome de Mirizzi, ainda que uma afecção pouco frequente, é uma das possibilidades etiológicas, descritas na literatura, para a elevação do marcador tumoral CA 19-9, e mesmo sendo encontrado até então, um número muito pequeno de relatos médicos, é um diagnóstico benigno para situações que cursam com elevação do antígeno carboidrato de superfície celular, que tem seu valor de referência normalizado após tratamento cirúrgico do processo obstrutivo. Dessa forma, ressalta-se a importância da descrição do caso aqui discutido. 


\section{REFERÊNCIAS}

1. BALLEHANINNA UK, CHAMBERLAIN RS. The clinical utility of serum CA 19-9 in the diagnosis, prognosis and management of pancreatic adenocarcinoma: An evidence based appraisal. Journal of Gastrointestinal Oncology, 2012; 3(2): 105-119.

2. FONTES PRO, et al. Síndrome de Mirizzi em associação com níveis séricos de CA 19-9 superiores a $20.000 \mathrm{U} / \mathrm{mL}$ : é possível? ABCD: Arquivos Brasileiros de Cirurgia Digestiva (São Paulo), 2012; 25(1): 69-70.

3. GIBOR U, et al. CA 19-9 in the Presence of Obstructive Jaundice due to Mirizzi Syndrome. The Israel Medical Association Journal, 2015;17(1): 60-61.

4. SHAH OJ, et al. Management of Mirizzi syndrome: a new surgical approach. ANZ Journal Surgery, 2001; 71: 423427.

5. SILVA JB, et al. Síndrome de Mirizzi associada à fístula colecistogástrica. Relatos Casos Cirúrgicos, 2016; (2): 1-3

6. JUNIOR EVM, et al. Mirizzi's syndrome:case report and literature review. GED, 2015; 14(1).

7. ENGLAND RE, MARTIN DF. Endoscopy Managernent of Mirizzi's Syndrorne. Gut, 2017; 40(2): 272-6.

8. ROBERTSON AG, DAVIDSON BR. Mirizzi syndrome complicating an anomalous biliary tract: a novel cause of a hugely elevated CA19-9. Eur Journal Gastroenterology Hepatology, 2007; 19(2): 167-69.

9. LAI ECH, LAU WY. Mirizzi Syndrome: history, present and future development. ANZ, J. Surgery, 2006; $76: 251$

10. IBRARULLAH M, et al. Mirizzi syndrome. Indian Journal of Surgery, 2008; 70: 281-287.

11. CSENDES A, et al. The Relationship of Mirizzi Syndrome and Cholecystoenteric Fistula: Validation of a Modified Classification. World Journal Surgery, 2008; 32: 2237-2243.

12. LACERDA PS, et al. Mirizzi syndrome: A surgical challenge. ABCD: Arquivos Brasileiros de Cirurgia Digestiva (São Paulo), 2014; 27(3): 226-227.

13. ROSENFELD LG, et al. Valores de referência para exames laboratoriais de hemograma da população adulta brasileira: Pesquisa Nacional de Saúde. Rev. bras. epidemiol., Rio de Janeiro , 2019; 22(2).

14. LEITE LMC, COELHO JF. Síndrome de Mirizzi: contribuição ao diagnóstico e à terapêutica. Radiologia Brasileira, 2006; 39(3): 226.

15. LIN CL, et al. Mirizzi's syndrome with a high CA19-9 level mimicking cholangiocarcinoma. Am J Gastroenterol, 1997; 92(12): 2309-10.

16. MANN DV, et al. Elevated tumour marker CA19-9: clinical interpretation and influence of obstructive jaundice. Eur Journal Surgery Oncology, 2000; 26(5): 474-79.

17. PRINCIPE A, et al. Mirizzi syndrome with cholecysto-choledocal fistula with a high CA19-9 level mimicking biliary malignancies: a case report. Hepato-gastroenterology, 2003; 50(53): 1259-62.

18. SANCHEZ M, et al. Elevated CA 19-9 levels in a patient with Mirizzi syndrome: case report. South Med Journal, 2006; 99(2):160-63. 